\title{
Combining SEM/EDS and Raman Microscopy for the Identification of Contaminant Particles in Pharmaceutical Products
}

\author{
P.R. Toepfer and G.L. Shearer
}

McCrone Associates, 850 Pasquinelli Drive, Westmont, IL 60559

Raman microscopy is becoming more popular as an analytical tool in a wide variety of industries and applications. While infrared microscopy is a more widely accepted technique for the identification of small particle contaminants, it requires time consuming sample preparation. Raman microscopy has several advantages over infrared including a smaller sample spot size for improved spatial resolution, an expanded spectral region and minimal sample preparation. Raman spectroscopy can be complicated by fluorescence with some samples, but this can sometimes be overcome using a longer wavelength excitation laser. Scanning electron microscopes with energy dispersive $\mathrm{x}$-ray spectrometry (SEM/EDS) systems are widely used to identify particles based on elemental composition, but are not specific for organic compounds. Sample preparation and choices of substrate can make phase identification difficult; for example, the identification of carbonates and oxides. Recently, a Raman spectrometer has been interfaced to SEM instruments to combine the morphological and elemental capabilities of SEM/EDS and the phase identification capabilities of Raman spectroscopy into a powerful tool for small particle identification. The SEM/SCA (Scanning Electron Microscope/Structural and Chemical Analyzer) (Renishaw) [1] allows for the analysis of a sample by both techniques from only one preparation; thus, reducing analysis time and minimizing the chance of loss, damage or alteration of the sample due to manipulation.

SEM imaging yields morphological information about particles. Using the SEM in backscatter imaging mode (BEI), one can quickly sift through a field of particles to focus on the particles composed of elements of a certain atomic number. For example, non-metallic particles of interest will stand out as dark against the brighter metallic particles. The BEI mode can also quickly show a "map" of composite particles that shows different phases of interest. Raman spectra can be obtained from various phases of the particle.

This paper will present a variety of examples to illustrate the advantages of the combined techniques. The particle shown in Fig. 1 is rich in silicon, but also has carbon present. However, the particle is mounted on a carbon sticky tab, so it is uncertain how much, if any, of the carbon is associated with the particle. The Raman spectrum of the particle clearly indicates that the silicon is present as silicon carbide as opposed to elemental silicon. A more complete phase identification of the particle aids in tracing the source of the contamination. In some cases, the EDS spectrum interpretation can be ambiguous. In Fig. 2, the spectrum of the particle indicates a silicate, possibly glass. The identification of glass in a pharmaceutical product is crucial. Glass particles often have a characteristic conchoidal morphology that is not obvious in this particle. The Raman spectrum is a good match for feldspar, a potassium aluminum silicate mineral.

Further examples and a discussion of sample substrate effects will also be discussed.

References

[1] A.D. Brooker et al., Imaging \& Microscopy 6 (2004) 48. 


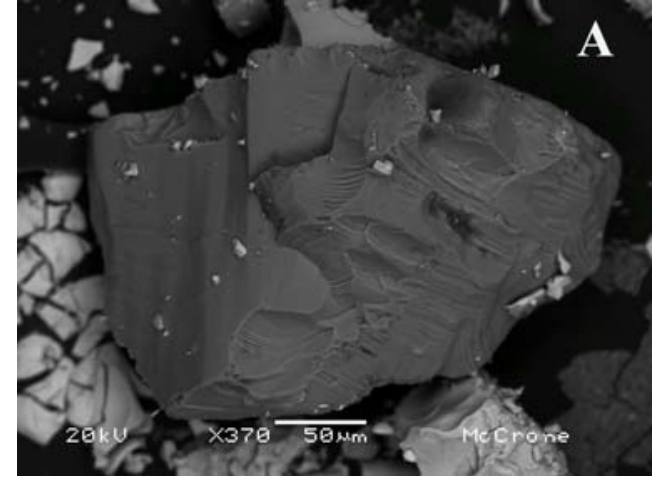

FIG. 1. The particle shown above (A) has high silicon and some carbon (B). The Raman spectrum of the particle $(\mathrm{C}$ - top) clearly indicates that it is silicon carbide ( $\mathrm{C}$ - bottom) and not elemental silicon.

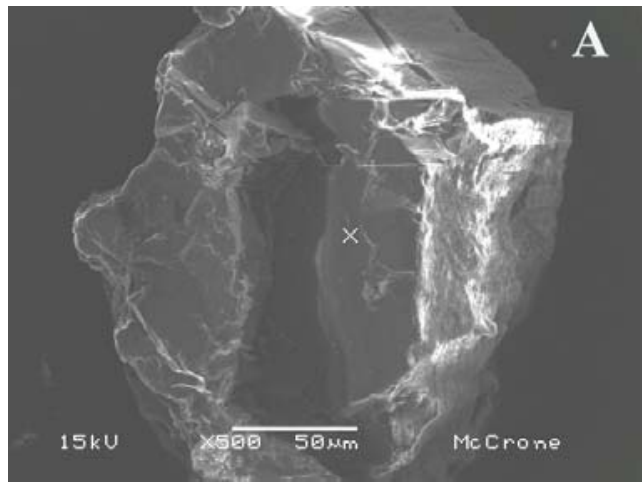

FIG. 2. The SEM image (A) above shows a particle with the elemental profile shown in B. The Raman spectrum of the particle ( $\mathrm{C}-$ top) is a good match for feldspar ( $\mathrm{C}$ - bottom).
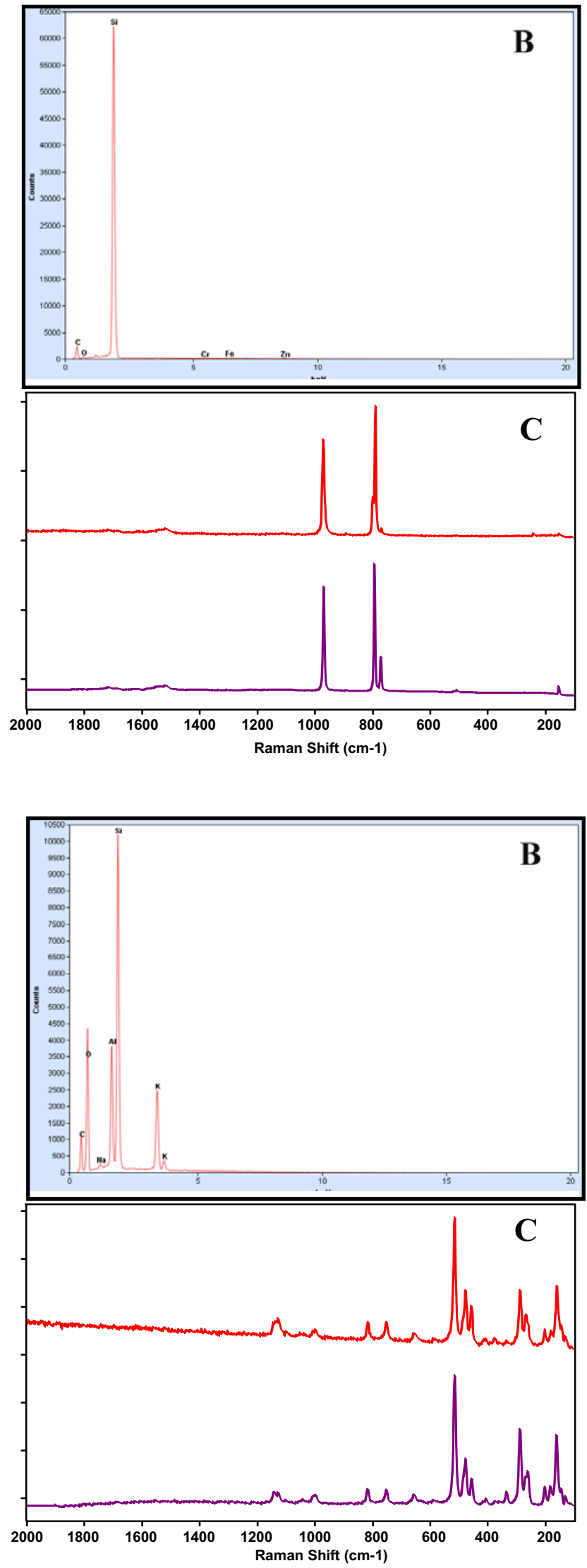